\title{
Los salvadoreños frente a las elecciones del 2000
}

El uso de las encuestas preelectorales ha venido en aumento en los últimos procesos electorales. De hecho, en cada elección-sea ésta presidencial, legislativa o municipal- existen más encuestas, scan éstas independientes o partidarias, que son publicadas y discutidas a través de los medios. Si de algo parece caracterizarse desde ya al proceso cleccionario del $2000 \mathrm{es}$ del intenso uso de las encuestas que se ha hecho por parte de todos los interesados en las elecciones. En la semana del 21 al 25 de febrero de 2000 , aparecieron públicamente no menos de seis encuestas, incluyendo dos que lucron patrocinadas por los dos principales partidos políticos de contienda.

La mayor parte de las encuestas y de la discusión pública sobre ellas se concentraron en adelanlar los posibles resultados de las elecciones legislativas a nivel nacional y, dada su importancia, en los posibles resultados de la elección municipal en San Salvador. Aunque no se puede negar el interés que genera este aspecto de las encuestas preelectorales, en realidad hay que decir que su contribución à un debate y a un análisis serio sobre los procesos electorales es mínimo. Su importancia no va más allá de la especulación sobre los posibles escenarios que resulten como producto de las elecciones, aunque ello puede hacerse fácilmente sin acudir al uso de los sondeos de opinión pública. De ahí que más allá de los posibles intereses que puedan tener los partidos políticos que hicieron públicas su propias encuestas, el discutir simplemente Ios posibles resultados partidistas empobrece la posible utilidad de las encuestas.
En el fondo, las encuestas realizadas un mes o inclusive quince días antes de las elecciones no pueden ser el instrumento más válido para adelantar quien ganará el proceso electoral. Después de todo, muchas cosas pueden ocurrir en ese lapso de tiempo desde que el trabajo de recolección de datos de la encuesta es terminado hasta que se celebran las votaciones; así como también, muchas personas dejan de contestar la encuestas escudándose en su derecho de no revelar su voto. En tal sentido, la validez fundamental de las encuestas electorales - no siempre comprendida por los medios de comunicación y por los analistas políticos- radica en que el hecho de que contribuyen a entender cuál es la realidad subjetiva que constituye el contexto del proceso electoral $y$, en tal sentido, ayudan a comprender por qué la gente se comporta electoral y políticamente de la manera en que lo hace.

La última encuesta del Instituto Universitario de Opinión Pública de la UCA fue planteada de esa forma y, por ello, toma distancia del resto de sondeos preelectorales dados a conocer en la última semana de febrero. El objetivo fundamental de la encuesta del IUDOP era comprender cómo se sienten los salvadoreños de cara al proceso electoral, de 2000 , por qué van a votar o no van a votar y por qué piensan votar apoyando a un partido y no a otro. El sondeo en cuestión se componía de dos muestras distintas. En primer lugar, se tomó una muestra nacional de 1233 adultos de todo el país -es decir, que residen en los catorce departamentos del país - tanto en áreas urbanas como rurales', sobre la base de una selección aleatoria por

1. La proporción de entrevistas realizadas en el área rural fue de sólo el 35 por ciento, las cuales posteriormente lueron ponderadas para darles el peso que corresponde a nivel nacional (45 por ciento). 
etapas basada en segmentos censales en los que fueron divididos todos los municipios seleccionados de cada uno de los departamentos. El error muestral de esta muestra fue de $+/-0.028$ (2.8 por ciento). En segundo lugar, se tomó una muestra para el municipio de San Salvador, consistente en 631 personas mayores de 18 años que residen en San Salvador y/o que habrán de votar en ese municipio. En este caso, la selección también se hizo sobre segmentos geográficos y con un error muestral de +/-0.04 (4 por ciento). Hay que decir que en ambas muestras se utilizó básicamente el mismo cuestionario, es decir, se hicieron las mismas preguntas, con la excepción de que en San Salvador se hicieron algunas preguntas adicionales referidas a las particularidades capitalinas.

Este breve artículo tiene por objetivo presentar un resumen de los hallazgos de la encuesta, adelantando de forma general algunas de las conclusiones que pueden extraerse de los mismos datos.

\section{El interés hacia las elecciones}

En términos generales, los salvadoreños parecen estar poco interesados en la realización de las elecciones municipales y legislativas del 2000. Más de la mitad de los consultados, el 57.2 por ciento de los salvadoreños, tiene poco o ningún interés en los comicios a celebrarse el próximo 12 de marzo. El estudio de opinión pública reveló que solamente el 22.6 por ciento de los salvadoreños se encuentran muy interesados en las elecciones y que el resto de personas llegan a sentir "algo" de interés por el proceso. Lo anterior sugiere que el interés por las elecciones mostrada por las élites políticas y por los medios de información del país no es compartido por la mayor parte de la población salvadoreña, especialmente por aquella de menores recursos socioeconómicos. Y es que de hecho, el porcentaje de personas que, en plena campaña electoral, se sienten muy interesadas por la política en general es bastante bajo (ver Cuadro 1). Solamente uno de cada diez salvadoreño tiene mucho interés por la política. Esto explica, en parte, el hecho de que sólo el 30.7 por ciento se ve muy motivado a votar, frente a un 21.5 por ciento que se muestra "algo" motivado para votar y un 47.8 por ciento que se expresó poco o nada interesado en asistir a votar. Elecciones anteriores han demostrado que al final sólo asiste la gente que se muestra muy motivada para votar.

\section{Cuadro 1 \\ Interés en la política, en las elecciones \\ y en asistir a votar \\ (En porcentajes)}

\begin{tabular}{lcccc}
\hline & \multicolumn{4}{c}{ Nivel } \\
\cline { 2 - 5 } Interés & Mucho & Algo & Poco & Nada \\
\hline En la política & 11.0 & 13.7 & 33.9 & 41.4 \\
En el proceso & 22.6 & 20.2 & 33.9 & 23.3 \\
En asistir a votar & 30.7 & 21.5 & 25.2 & 22.6 \\
\hline
\end{tabular}

¿Cuánto le interesa la política?/ ¿Cuánto le interesa el proceso electoral?/¿Qué tanto está usted interesado en ir a votar el 12 de marzo?

Además, los resultados muestran que sólo el 30 por ciento de los ciudadanos creen que las elecciones serán limpias. El resto de los salvadoreños piensan que habrá fraude ( 41.4 por ciento) o simplemente no saben si las elecciones serán transparentes (28.6 por ciento).

La encuesta de la UCA encontró nuevamente que a la base de estas actitudes hacia las elecciones se encuentran tres grandes grupos de opiniones sobre la política y el país que también están muy relacionadas entre sí. En primer lugar, la mayor parte de los salvadoreños piensa que los partidos políticos no les benefician ni tampoco les representan adecuadamente. En concreto, el 63.6 por ciento estuvo en desacuerdo con la idea de que los partidos políticos benefician a la población; mientras que, por otro lado, el 59.2 por ciento considera que los partidos políticos no representan los intereses de los salvadoreños. En segundo lugar, detrás del poco afecto de los salvadoreños por las elecciones existe la impresión de que las mismas no son útiles para cambiar y mejorar las cosas en el país. De hecho, el 54 por ciento de los consultados dijeron que las elecciones son una pérdida de tiempo pues las cosas nunca cambian en el país. Esta opinión es particularmente grave entre una ciudadanía en la que prevalece la percepción (69.5 por ciento) de que la situación del país está empeorando.

Finalmente, el sondeo realizado por el IUDOP indica que buena parte de la apatía hacia las elecciones está profundamente vinculada a la desconfianza ciudadana en las instituciones políticas del país y en la gestión del gobierno central (lo cual 
también explica parte de las intenciones de voto). La mayor parte de las instituciones políticas del país, con excepción de las alcaldías, no reciben más allá del 15 por ciento de confianza absoluta por parte de los salvadoreños. Instituciones como el gobierno, la Asamblea y, sobre todo, los partidos políticos han recibido los menores índices de confianza ciudadanos (ver Cuadro 2).

\section{Cuadro 2 \\ Confianza en algunas instituciones del país (En porcentajes)}

\begin{tabular}{lccccc}
\hline Institución & Mucha & $\begin{array}{c}\text { Confianza } \\
\text { Algo }\end{array}$ & Poco & Nada & No responde \\
\hline Alcaldía local & 15.3 & 22.3 & 33.9 & 27.2 & 1.3 \\
Tribunal Supremo Electoral & 10.7 & 19.9 & 33.8 & 31.1 & 4.5 \\
Gobierno central & 8.0 & 17.1 & 35.4 & 37.1 & 2.4 \\
Asamblea Legislativa & 4.0 & 14.1 & 40.2 & 38.1 & 3.6 \\
Partidos políticos & 3.8 & 15.1 & 38.9 & 41.3 & 0.9 \\
\hline
\end{tabular}

Ahora le voy a pedir que me diga cuánta confianza tiene usted en las instituciones que le voy a mencionar.

Opiniones parecidas se reflejan también cuando se trata de evaluar concretamente el desempeño de algunas instituciones. Cuando se trata de valorar su gestión, el gobierno de Francisco Flores fue calificado con una nota promedio de 4.84 (la más baja en todos los mandatos de ARENA), mientras que la Asamblea Legislativa recibió una nota media aún menor: 4.74. Sólo los gobiernos municipales, en su conjunto nacional, parecen recibir menos críticas de la población, 5.92, aunque el promedio sigue siendo bajo ${ }^{2}$. Estas evaluaciones sobre el desempeño de las instancias del gobierno no sólo afectan a los partidos directamente involucrados sino también a todo el sistema político electoral dado que, decepcionados de sus propios gobernantes, buena parte de los ciudadanos se retiran de la posibilidad de ejercer su volo.

\section{Las preferencias partidarias y las intenciones de voto}

En este contexto de mucho desinterés y desconfianza ciudadana, el sondeo realizado por la UCA revela que más de la mitad de los salvadoreños no se sienten simpatizantes de ningún partido político en contienda y que la identificación partidaria por cada uno de los partidos no supera al 18 por ciento de toda la población adulta en edad de votar. Para febrero del 2000 , el porcentaje de personas que se identifican con ARENA es del 17 por

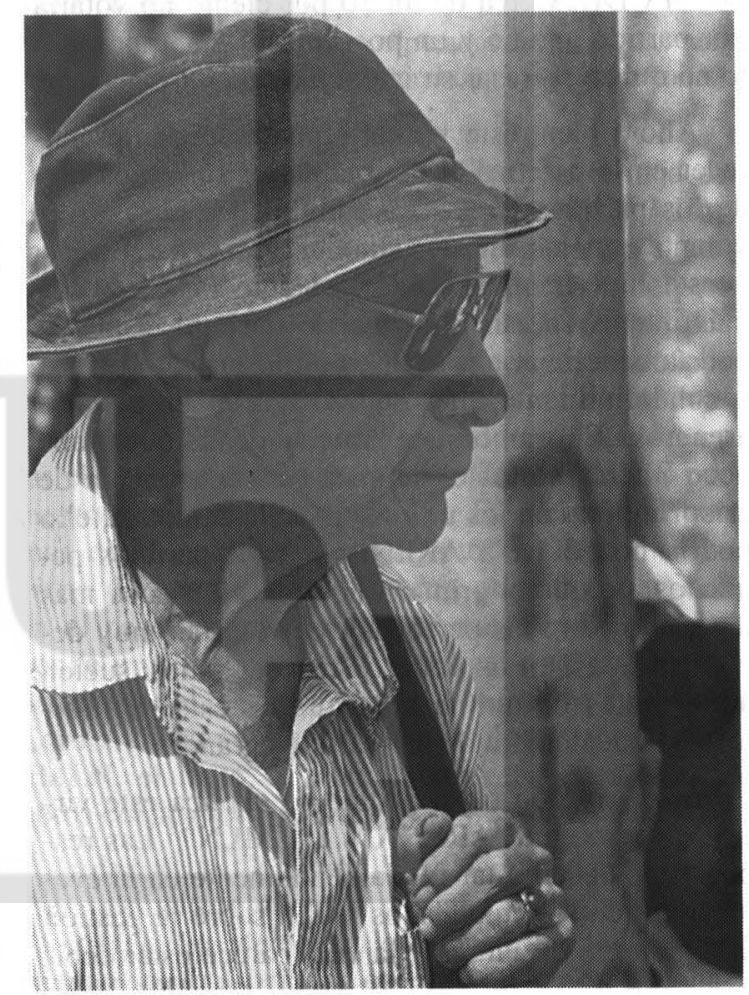

2. Sin embargo. los gobiernos municipales de San Salvador y de Antiguo Cuscatlán fueron los mejor evaluados por sus pobladores. 
ciento; mientras que el FMLN reúne al 14.6 por ciento de todos los adultos del país; el PDC logra una identificación partidaria del 2.8 por ciento; en tanto que el PCN y el CDU logran el 2.2 y el 1.3 por ciento respectivamente. El resto de partidos juntos no logran más allá del 3 por ciento.

Ahora bien, la encuesta mostró que no siempre la gente parece estar dispuesta a votar en función de su simpatía partidaria. El estudio de opinión realizado por el IUDOP de la UCA revela un virtual empate en la intención de voto para diputados a nivel nacional. Lo cual significa que algunas de las preferencias partidarias se han desplazado o que algunas de las personas que no tienen partido de preferencia tienen intenciones de apoyar con su voto a un partido político.

Para mediados de febrero, el 21.8 por ciento de las personas que tienen carnet electoral dijeron que votarian por ARENA; el 21.6 por ciento dijo que votaría por el FMLN; el 4.2 por ciento sostuvo que votaría por el $\mathrm{PCN}$; el 3 por ciento afirmó que votaría por el PDC y el 2.5 por ciento votaría por el CDU. Cerca de un 10 por ciento no votaría por partido alguno y un poco más del 35 por ciento no quiso expresar su intención de voto.

Ahora bien, este virtual empate puede desaparecer en la práctica en la medida en que cada uno de los partidos tenga más habilidades para atraer votantes en los últimos días de campaña. La encuesta muestra que si al final sólo la gente con más interés en asistir a votar lo hace, el resultado podría beneficiar al FMLN, porque su voto parece ser más militante, más seguro que el de muchos areneros. En cambio, si mucha gente que tiene poco interés en las elecciones está convencida de asistir a votar en los últimos días de campaña, ello podría beneficiar a ARENA y a los partidos pequeños, porque el primero encuentra un poco más de apoyo entre las personas que no están muy decididas a asistir a votar y los segundos suelen atraer votos de última hora que no están conformes con cualquiera de los partidos grandes.

Pero, en todo caso, ¿cómo se explica este virtual empate entre ARENA y el FMLN?? Las respuestas parecen estar fundamentalmente en la evaluación que los ciudadanos hacen sobre la gestión del gobierno de Flores y la cual parece estar debi- litando a ARENA y contribuyendo indirectamente al FMLN y al PCN. Los resultados de la encuesta de la UCA indican que las únicas personas que aprueban la gestión presidencial -es decir, los que evalúan con promedio arriba de 6- son los electores de ARENA, el resto de personas independientemente de su afiliación partidista, no aprueba la gestión ejecutiva.

\section{Cuadro 3}

Preferencia partidista e intención de voto nacional para diputados (En porcentajes)

\begin{tabular}{|c|c|c|}
\hline Partido & Preferencia & $\begin{array}{c}\text { Intención de } \\
\text { voto diputados* }\end{array}$ \\
\hline Ninguno & 55.6 & 9.5 \\
\hline ARENA & 17.0 & 21.8 \\
\hline FMLN & 14.6 & 21.6 \\
\hline PDC & 2.8 & 3.0 \\
\hline $\mathrm{PCN}$ & 2.2 & 4.2 \\
\hline CDU & 1.3 & 2.5 \\
\hline PAN & 0.4 & 0.5 \\
\hline USC & 0.3 & 0.4 \\
\hline PLD & 0.2 & 0.4 \\
\hline No sabe, secreto & 5.5 & 36.1 \\
\hline
\end{tabular}

¿Cuál es el partido político de su preferencia?/ ¿Por cuál partido votaría Ud. para diputados?

* La intención de voto para diputados incluye a las personas que tienen carnet electoral o que está en trámite.

De hecho, ARENA parece estar dependiendo más de su voto duro y de votantes nuevos jóvenes, que de antiguos correligionarios de otros partidos. Por su parte, el FMLN parece estar ganando un poco más de adeptos entre las personas que votaron para otros partidos en las elecciones de 1999 , especialmente de ARENA. El desplazamiento desde ARENA no sólo se da hacia el FMLN sino también hacia el PCN y hacia un grupo importante de posibles abstencionistas electorales.

Por otro lado, la UCA preguntó a la población sobre cuál es el tipo de Asamblea que le conviene más al país. Alrededor del 40 por ciento de los salvadoreños cree que al país le conviene una Asamblea en la que no domine partido político al-

3. Entre los que tienen carnet electoral o muy probablemente lo tengan para el día de las elecciones. 
guno, es decir en la que haya equilibrio de todas las fuerzas; un 14.5 por ciento considera que la Asamblea debería ser dominada por ARENA; un 9.1 por ciento cree que debería ser dominada por el FMLN y un 11.4 por ciento otorga relevancia a los partidos pequeños; el resto de personas no supo o no quiso responder a la pregunta. En términos generales, estas opiniones parecen desfavorecer más a ARENA que a cualquier otro partido político, lo cual en el fondo beneficia indirectamente al FMLN y al PCN, pues el partido oficial es visto como el partido al cual hay que restar peso. Lograr el equilibrio que implica que ningún partido domine la Asamblea es apoyar a otros partidos que no sean el partido de gobierno; en tanto que beneficia al FMLN porque es visto como el principal contendiente del gobierno y al PCN porque constituye una opción ideológica afín sin tener que votar por el oficialismo.

La intención de voto para la alcaldía de San Salvador

Por la importancia que tiene el gobierno municipal de San Salvador, el IUDOP decidió realizar una muestra aparte de 631 entrevistas para el municipio de San Salvador con el objeto de explorar las actitudes de la población con respecto a las elecciones municipales.

En primer lugar, los residentes en el municipio de San Salvador muestran un nivel de interés relativamente mayor por las elecciones que en el resto del país. De acuerdo a los resultados, un $\mathbf{4 0 . 1}$ por ciento de los capitalinos estarían muy interesados en asistir a votar el próximo 12 de marzo. En segundo lugar, las intenciones de voto para el municipio de San Salvador favorecen ampliamente a Héctor Silva y al FMLN. De acuerdo a los resultados, el 40.2 por ciento de los residentes del municipio de San Salvador que posee carnet electoral votaría por la Coalición FMLN-USC, seguidos de un 23.1 por ciento que votaría por ARENA, un 2 por ciento que votaría por el PDC y un 1.8 por ciento que elegiría al resto de partidos. Sin embargo, un poco más del 25 por ciento no reveló su intención de voto y casi el 7 por ciento dijo que no votará por partido alguno.

En cuanto a la simpatía por el candidato, los resultados confirman la ventaja del candidato de la Coalición FMLN-USC. Héctor Silva recibe el apoyo del 53.2 por ciento de los capitalinos, en tanto que Luis Cardenal recibe el apoyo del 23.1 por ciento. El resto de candidatos no recibe más del 4 por ciento de las simpatías capitalinas y un poco más del 20 por ciento restante no define su candidato de simpatía.

\section{Cuadro 4 \\ Intención de voto y candidato preferido para la Alcaldía de San Salvador (En porcentajes)}

\begin{tabular}{lrc}
\hline Partido & $\begin{array}{c}\text { Intención de } \\
\text { voto* }\end{array}$ & $\begin{array}{c}\text { Candidato } \\
\text { preferido }\end{array}$ \\
\hline Ninguno & 6.8 & 7.5 \\
FMLN-USC/Héctor Silva & 40.2 & 53.3 \\
ARENA/Luis Cardenal & 23.8 & 23.1 \\
PDC/Ricardo Martínez & 2.0 & 1.0 \\
PCN/Marta Castro & 0.5 & 0.7 \\
Otros partidos/Otros & 1.3 & 1.8 \\
candidatos & 25.4 & 13.0 \\
No sabe, secreto & \\
\hline
\end{tabular}

¿Por cuál partido votaría Ud. para alcalde?/¿Quién es el mejor candidato a la alcaldía de San Salvador?

* La intención de voto para diputados incluye a las personas que tienen carnet electoral o que está en trámite.

¿Cómo se explica esa considerable ventaja de Héctor Silva y la Coalición sobre ARENA? Hay muchas razones, pero la encuesta registra un significativo desplazamiento hacia la Coalición de personas que en las elecciones de 1999 votaron por ARENA y otros partidos. En concreto, el 16 por ciento de los que piensan votar a favor de la Coalición en el 2000, votaron apoyando a ARENA en las elecciones anteriores y un poco más del 10 por ciento voló por otros partidos. Esto significa que más de la cuarta parte del voto de Héctor Silva viene de personas que votaron por otros partidos en el pasado reciente. Por su parte, ARENA depende mucho más de su voto leal que el FMLN. Solamente el 6 por ciento del voto a favor de Luis Cardenal viene de otros partidos, incluido el FMLN, el resto de votos son votantes nuevos o votos constantes de ARENA (64.2 por ciento).

Además, la Coalición FMLN-USC parece estar logrando sumar adeptos por efecto de la gestión capitalina. En efecto, en este caso, las tendencias parecen estar asociadas con la valoración que hacen los capitalinos sobre el desempeño del gobierno municipal. Los votantes del bloque FMLN-USC acusan un elevado promedio de evaluación a la ges- 
tión del alcalde Silva y el resto de simpatizantes de otros partidos, con excepción de ARENA y el PCN, suelen darle también una calificación positiva. Ello estaría teniendo más peso a la hora de decidir el voto que la propaganda del partido oficial.

\section{Resumen}

La última encuesta preelectoral del Instituto Universitario de Opinión Pública de la UCA revela que, al igual que los procesos electorales anteriores, la mayor parte de la gente no está interesada ni motivada por las elecciones legislativas y municipales del 2000 . A la base de esta apatía parecen encontrarse varios factores. Por un lado, la ya inveterada percepción de que lo político no es de utilidad para mejorar las condiciones de vida de la mayoría de la población; por otro lado, la sensación popular de que el país está cada más emproblemado; y, en tercer lugar, la poca credibilidad que se han forjado la mayor parte de instituciones políticas del país y el desencanto provocado por el desempeño del gobierno y de la Asamblea Legislativa.

Lo más relevante de la encuesta de la UCA no está en la predicción de las intenciones de voto partidario, sino en el hecho de que muestra cómo el sistema político salvadoreño sigue siendo incapaz de conectar efectivamente con los anhelos y preocupaciones más importantes de los ciudadanos y cómo esto tiene un impacto negativo en la participación electoral.
Los resultados de la encuesta muestran un virtual empate entre ARENA y el FMLN en la disputa por los diputados a nivel nacional, mientras que el resto de partidos acumulan preferencias residuales de parte de la población. El desenlace final de las elecciones dependerá del grado de participación ciudadana, la cual podría beneficiar a un partido y desfavorecer a otro. En la práctica es probable también que los partidos pequeños obtengan mejores resultados que los que aparecen en las encuestas, aunque sin llegar a representar grandes sorpresas.

Con todo y sin negar la posible influencia propagandística, hay que decir de que el resultado de las elecciones parlamentarias depende también de lo que el gobierno central y las alcaldías hayan hecho o hayan dejado de hacer durante su gestión.

Por su parte, la disputa por la alcaldía de San Salvador favorece ampliamente a la Coalición y especialmente al candidato Silva. Este caso y el de otras municipalidades de cualquier signo político es un ejemplo de cómo una gestión valorada positivamente por la mayoría de los ciudadanos puede no sólo atraer más votos inusuales a un partido político, sino que también puede beneficiar al sistema político porque fomenta el interés ciudadano por la participación y la competitividad electoral.

\section{Instituto Universitario de Opinión Pública}

